\title{
Editorial Impression on the Cardiology Section of Aged Human Suffering from Aortal Blockage by Heavy Smoking
}

\section{Vincent John*}

Department of Gynaecology and Health Sciences, Faculty of Medicine, Universidade de Sao Paulo, Argentina

*Corresponding author: Vincent John, Department of Gynaecology and Health Sciences, Faculty of Medicine, Universidade de Sao Paulo, Argentina, Tel +5511 3091-3116; E-mail: vincentjohnvj@rediffmail.com

Received date: June 28 2017, Accepted date: July 03 2017, Published date: July 052017

Copyright: 2017 (c) John V. This is an open-access article distributed under the terms of the Creative Commons Attribution License, which permits unrestricted use, distribution, and reproduction in any medium, provided the original author and source are credited.

\section{Editorial}

Most of the aged and fatty person are going to be initialized in the open heart surgery because of their improper functioning of heart, arteries, valves etc... this kind of probability is seen in mainly smokers, junk food eaters, rarely genetic. BYPASS surgery for these type of people is not only risk but also endangerous in late ages, heavy bodies. After getting successful surgery reports, with in short time some of them are dying because they are not willing to with draw their past habits and habitats.

\section{Open heart cardio surgery necessity and its image clinically}

One of the cardiologic survey reports that patients who keep on smoking until the date of remedial expectation lung disease surgery have a higher postoperative dismalness, including higher recurrence of PPC, longer clinic LOS, and a higher recurrence of ITU confirmation. In ex-smokers there was incline for lessened recurrence of PPC and ITU confirmation contrasted with current smokers, yet there was no huge distinction in watched result between patients who quit smoking less $<6$ weeks versus $\geq 6$ weeks before surgery. We found no noteworthy contrasts in early mortality or long haul survival between any of the gatherings inside our subsequent period. Our think about showed that the frequency of PPC in current smokers is $29 \%$, which is more noteworthy than 10 times that of never smokers and twice that of exsmokers (Figure 1).

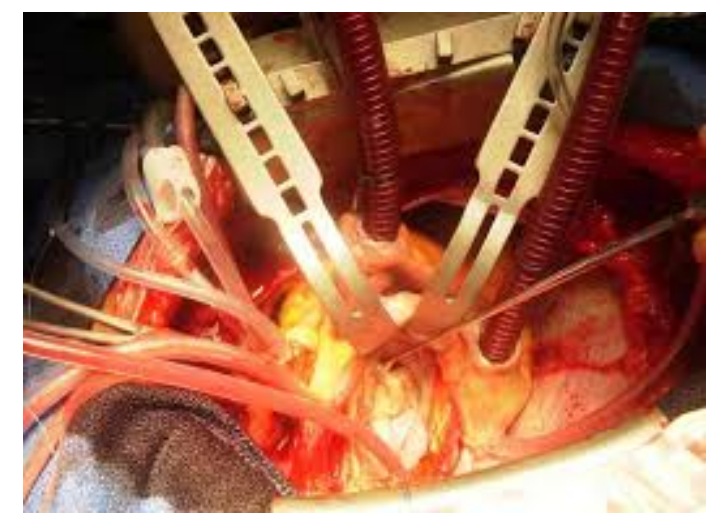

Figure 1: BYPASS Module and supplying blood transfusion, pumping.

Contrasting this watched occurrence and results from different examinations is testing a result of the distinctions in the meaning of PPC, which may reach out to incorporate conceivably more extreme yet less regular entanglements, for example, pneumonic embolism, delayed air spills and bronchopleural fistulas. Regardless of this, the recurrence of PPC in smokers utilizing the MGS is inside the extensive variety of aspiratory occasions announced in different examinations (7.3-39.7\%). Our observing of PPC to be higher in current versus never smokers is additionally upheld by different investigations. Smoking had no immediate impact on postoperative mortality or long haul survival amid in our investigation. By and by, the balanced survival bends for never smokers were obviously over those of the other smoking classes particularly after the initial 16 months of development, proposing a feasible more positive result despite the fact that this did not achieve measurable hugeness. This might be to some degree because of the short follow up period or the smoking propensities for patients after surgery; some may stop while others may restart. Different examinations have demonstrated expanded mortality in smokers contrasted with never smokers, however no critical distinction among the current and ex-smoking gatherings. The injurious impact of kept smoking on long haul survival subsequent to being determined to have lung disease is progressively apparent, yet few papers have investigated the impact of preoperative smoking suspension on long haul survival in lung tumor patients experiencing remedial plan surgery. A multicenter audit of 247 patients experiencing NSCLC resection found that present smokers had a diminished 5-year survival in contrast with never smokers (68\% versus $86.5 \%, \mathrm{p}=0.06$ ), and that ceasing smoking a year before disease determination had no noteworthy distinction in long haul survival. Strangely, albeit smoking was not a free hazard consider for latepassing's our examination, PPC improvement and LOS were autonomous hazard elements for late-passing's, both of which were essentially expanded in the present smokers, here provided the death rate, risk factor in heavy moment Table 1.

\begin{tabular}{|l|l|l|l|l|l|}
\hline Variables & $\begin{array}{l}\text { Parameter } \\
\text { estimate }\end{array}$ & SE & p-value & HR & $\mathbf{9 5 \%} \mathbf{C I}$ \\
\hline Stage (II vs. I) & 1.11 & 0.22 & $<0.0001$ & 3.03 & $1.97-4.71$ \\
\hline Stage (IIIA vs. I) & 1.35 & 0.25 & $<0.0001$ & 3.87 & $2.39-6.28$ \\
\hline Stage (IIIB vs. I) & 1.88 & 0.61 & 0.0019 & 6.54 & $1.57-18.33$ \\
\hline Age & 0.03 & 0.01 & 0.0115 & 1.03 & $1.01-1.05$ \\
\hline PPC & 0.53 & 0.27 & 0.0469 & 1.71 & $0.98-2.82$ \\
\hline LOS & 0.03 & 0.01 & 0.0324 & 1.03 & $1.01-1.05$ \\
\hline
\end{tabular}

Table 1: Independent risk factors for late deaths. 
Citation: Vincent J (2017) Editorial Impression on the Cardiology Section of Aged Human Suffering from Aortal Blockage by Heavy Smoking. Med Rep Case Stud 2: e102. doi:10.4172/2572-5130.1000e102

Page 2 of 2

One of the liberal report revealed in an overall word around 10 in 50 patients keep on smoking preceding corrective purpose surgery for NSCLC. Smoking suspension preceding surgery decreases PPC frequency and also other critical markers of postoperative grimness, along these lines we suggest that all patients ought to experience formal smoking end as business as usual work up. The ideal planning for preoperative smoking end however is yet to be characterized.
Additionally explore into compelling preoperative smoking suspension programs and their here and now and long haul impacts is critically required. The vast majority who've had a CABG advantage from taking an interest in an organized, far reaching restoration program. This is generally done outpatient with visits a few times each week. The parts to the program incorporate exercise, decreasing danger components, and managing stress, nervousness, and despondency. 